\title{
Répercussion des rabais sur les factures
}

\section{Patrick Müller}

Chef de division, division Médecine et tarifs ambulatoires, FMH

\begin{abstract}
Les fournisseurs de prestations doivent répercuter sur le débiteur de la rémunération les avantages directs ou indirects qu'ils perçoivent. Ni la loi ni l'ordonnance concernées n'indiquent cependant comment les rabais doivent figurer sur les factures. La FMH a rédigé une recommandation pour que les rabais soient répercutés de manière transparente et conforme à la loi, et qu'ils figurent sur les factures.
\end{abstract}

Après l'entrée en vigueur des nouveaux art. 55 et 56 de la loi sur les produits thérapeutiques (LPTh) le 1 $^{\text {er }}$ janvier 2020 , la question de «l'obligation de répercuter les rabais et ristournes» a connu un regain d'intérêt alors que depuis 1996, elle était réglée de manière générale par l'art. 56 al. 3 de la loi sur l'assurance-maladie (LAMal). La LAMal fixe le principe de l'obligation de répercuter intégralement les avantages ${ }^{1}$. Le fournisseur de prestations doit répercuter sur le débiteur de la rémunération les avantages directs ou indirects, tels que les rabais ou les ristournes (art. 56 al. 3 LAMal). Dans le système du tiers garant, les débiteurs sont les patients, dans celui du tiers payant, les assureurs-maladie. Les fournisseurs de prestations ne peuvent donc pas garder pour eux les avantages qu'ils perçoivent (interdiction des avantages/devoir d'intégrité). Plus exactement, ils ont l'obligation de répercuter les éventuels rabais qui leur sont accordés pour des médicaments ou des dispositifs médicaux (obligation de répercussion).

\section{Transparence et clarté}

L'art. 56 al. 3bis LAMal définit des exceptions à cette obligation lorsque certaines dispositions sont respectées. Ces exceptions exigent toutefois une convention préalable entre les fournisseurs de prestations et les assureurs et, pour l'heure, elles ne sont possibles que dans le cadre de l'art. 56 al. 3 let. b LAMal - à savoir en lien avec la vente ou l'achat de médicaments ou de moyens et d'appareils diagnostiques ou thérapeutiques. De plus, ces conventions doivent remplir les conditions suivantes: premièrement, garantir qu'une majeure partie (et non l'intégralité) des avantages sera répercutée, c'est-à-dire une part supérieure à 50\% et inférieure à $100 \%$, et, deuxièmement, que les avantages non répercutés seront utilisés de manière vérifiable exclusivement pour améliorer la qualité du traitement. La FMH a rédigé avec la communauté d'achat HSK (Helsana, Sanitas, KPT) et la CSS Assurance une convention-cadre réglant les principes fondamentaux en vue de la mise en œuvre de ces nouvelles dispositions.

La loi et l'ordonnance qui encadrent cette obligation ne précisent cependant pas comment les rabais doivent figurer concrètement sur les factures. Il n'existe pas non plus de recommandations communes des partenaires tarifaires sur la manière de reproduire sur les factures les accords économiques entre acteurs, qui, en pratique, prennent des formes très différentes. Une des exigences est assurément de veiller à la transparence et à la clarté.

Dans ce contexte, la FMH a rédigé une recommandation de mise en œuvre pour que les rabais accordés figurent de manière transparente (selon l'art. 42 LAMal) sur les factures et puissent être répercutés sur le débiteur de la rémunération. La recommandation montre les différentes manières de les répercuter et donne une vue d'ensemble des domaines concernés (médicaments, dispositifs médicaux). Par souci de transparence, de clarté et d'une meilleure compréhension, la FMH conseille donc aux médecins d'indiquer les rabais comme décrit ci-après, indépendamment du fait que les factures soient adressées aux patients (tiers garant) ou aux assureurs (tiers payant). 


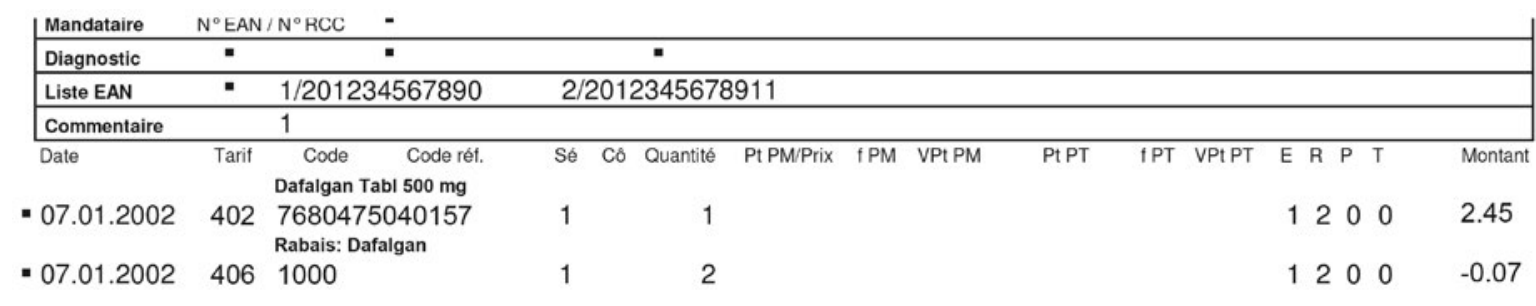

Figure 1: Exemple de facturation d'un médicament avec un rabais accordé de $5 \%(0,07$ franc) sur le prix de fabrique (1,33 franc); représentation de la $\mathrm{FMH}$.

2 Sur la base de la convention-cadre FMH/ HSK-CSS, convention qualité selon l'art. 56 al. 3bis LAMal.

\section{FMH}

Division Médecine et tarifs ambulatoires Baslerstrasse 47 CH-4600 Olten Tél. 0313591230 Fax 0313591238 tarife.ambulant[at]fmh.ch

\section{Mise en œuvre: recommandation de la FMH}

La FMH recommande d'ajouter sur les factures, d'ici fin 2022 au plus tard ${ }^{2}$, une position spécifique pour les rabais et les ristournes en utilisant le tarif 406 et le code 1000 "Rabais». Sur la facture, vous indiquez d'abord le dispositif médical ou le médicament concerné de manière usuelle avec son montant initial (prix public de la liste des spécialités par exemple). Ensuite, vous créez une deuxième position avec le tarif 406 , code 1000 , dans laquelle vous reportez le rabais effectif en francs sans mentionner le pourcentage accordé et vous pouvez adapter le texte de cette position en fonction de la situation. Le rabais doit être mentionné ainsi séparément pour chaque produit concerné (le code 1000 peut être utilisé plusieurs fois).

La figure 1 montre l'exemple d'un médicament sur un formulaire de facturation standardisé. Le médicament est facturé selon le prix public de la liste des spécialités, auquel est soustrait le rabais sur le prix de fabrique (indiqué en chiffre négatif sur la facture) au moyen de la position 1000 «Rabais».

\section{Les avantages de la recommandation de la FMH}

La FMH a essayé de trouver un accord concernant cette recommandation avec les assurances-maladie, qui souhaitent cependant privilégier une solution utilisant le facteur d'échelle (facteur externe). La FMH s'oppose énergiquement à cette méthode et recommande la solution avec le tarif 406 et le code 1000 «Rabais». La recommandation de mise en œuvre élaborée par la
FMH est préférable pour plusieurs raisons. Elle permet notamment d'établir une facture transparente, claire et facile à déchiffrer, y compris pour les patients.

En sa qualité d'association professionnelle des médecins en Suisse, la FMH accorde beaucoup d'importance à ce que l'ordonnance sur l'intégrité et la transparence dans le domaine des produits thérapeutiques (OITPTh) soit appliquée correctement et souhaite garantir la transparence et la clarté pour tous. A ce titre, elle estime qu'une position spécifique s'y prête de manière optimale et qu'il est facile d'en assurer le suivi (monitorage). Sur la facture, la distinction entre prix originaux et rabais permet de visualiser immédiatement le montant des avantages et de mettre en lumière la contribution des médecins et des fournisseurs aux économies dans le domaine de la santé. De cette manière, ces économies sont non seulement représentées de manière transparente sur chaque facture mais elles peuvent également être agrégées et analysées pour l'ensemble des médecins du secteur ambulatoire.

\section{Informations complémentaires}

Si vous avez des questions relatives à cette recommandation de la FMH, le Service juridique (lex[at]fmh.ch) ou la division Médecine et tarifs ambulatoires (tarife. ambulant[at]fmh.ch) se feront un plaisir de vous renseigner. Vous trouverez également des informations sur le site internet de la FMH: www.fmh.ch > Prestations > Droit > Ordonnance sur l'intégrité et la transparence dans le domaine des produits thérapeutiques (OITPTh). La recommandation de mise en œuvre est également accessible sur le site internet de la FMH. 